\title{
Dirichlet Form Approach to Infinite-Dimensional Wiener Processes with Singular Interactions
}

\author{
Hirofumi Osada \\ Department of Mathematical Sciences, University of Tokyo, 7-3-1 Hongo, Tokyo, 113 Japan. \\ e-mail: hi-osada@tansei.cc.u-tokyo.ac.jp
}

Received: 24 November 1994/in revised form: 9 June 1995

Dedicated to Professor Masatoshi Fukushima on his 60th birthday

\begin{abstract}
We construct infinite-dimensional Wiener processes with interactions by constructing specific quasi-regular Dirichlet forms. Our assumptions are very mild; accordingly, our results can be applied to singular interactions such as hard core potentials, Lennard-Jones type potentials, and Dyson's model. We construct nonequilibrium dynamics.
\end{abstract}

\section{Introduction}

Infinite-dimensional Wiener processes with interactions are diffusion processes with state space $\left(\mathbb{R}^{d}\right)^{\mathbb{N}}$ (or $\Theta$, where $\Theta$ is the set of all locally finite configurations of particles on $\mathbb{R}^{d}$ ) with interactions. When interactions come from a smooth pair potential $\Phi$ and martingale terms have constant coefficients 1 , these processes are described by the following SDE;

$$
d X_{t}^{i}=d B_{t}^{i}-\sum_{j=1, j \neq i}^{\infty} \frac{1}{2} \nabla \Phi\left(X_{t}^{i}-X_{t}^{j}\right) d t \quad(1 \leqq i<\infty),
$$

where $B_{t}^{i}(1 \leqq i<\infty)$ are independent Brownian motion on $\mathbb{R}^{d}$, and $\Phi: \mathbb{R}^{d} \rightarrow \mathbb{R}$. The associated $\Theta$-valued process is

$$
\mathbb{X}_{t}=\sum_{i=1}^{\infty} \delta_{X_{t}^{l}} \quad\left(\delta_{a} \text { is the delta measure at } a .\right)
$$

The study of (0.1) has been initiated by Lang [La1,2]. He solved (0.1) under suitable conditions on interactions for a set of initial configurations. Shiga [Sh] completed a gap of Lang's proof. Initial configurations for which (0.1) is solved were specified by Lippner [Li] and Rost [Ro] for $d=1$, and Fritz [F] for $d \leqq 4$.

Since Lang used SDE approach, a smoothness of $\Phi$ was crucial. He assumed:

(L.1) $\Phi \in C_{0}^{3}\left(\mathbb{R}^{d}\right)$, that is, $\Phi$ is finite range and of class $C^{3}$.

(L.2) $\Phi$ is super stable in the sense of Ruelle.

As a consequence some interesting examples were excluded. 
The purpose of this paper is to construct infinite-dimensional Wiener processes with interactions by constructing specific quasi-regular Dirichlet forms on a space $\Theta$ of configurations of infinitely many particles. The forms are governed by probability measures $\mu$ on $\Theta$ representing interactions of particles. We restrict our attention to $\Theta$-valued processes like $(0.2)$. One might construct labeled particle dynamics $(0.1)$ from $\Theta$-valued processes $(0.2)$ as functionals of $\mathbb{X}_{t}$. We will not pursue this here.

The advantage of our approach is the following: 1) Our assumptions are quite mild and weaker than Lang's assumptions. In our situation $\nabla \Phi$ may be distributions. In particular our results can be applied to singular interactions such as hard core potentials, Lennard-Jones 6-12 potentials, and Dyson's model [Sp2]. 2) Nonequilibrium dynamics can be constructed in the sense that we construct a family of diffusion measures $\left\{\mathbb{P}_{\theta}\right\}_{\theta \in \Theta}$ starting from each $\theta \in \Theta$; however, the uniqueness of diffusion $\left\{\mathbb{P}_{\theta}\right\}_{\theta \in \Theta}$ for a given equilibrium state $\mu$ holds only except for a set $\mathscr{N}_{\mu}$ of capacity zero. See also Remark (4) (stated after Theorem 3) about a remaining problem of identification in Lang's case.

We begin with some notations. A Radon measure $\theta$ is called a locally finite configuration if $\theta$ is of the form $\theta=\sum_{n} \delta_{x_{n}}$. Here $\left\{x_{n}\right\}$ is a finite or infinite sequence in $\mathbb{R}^{d}$ with no cluster points and $\delta_{a}$ is the delta measure at $a$. By convention we regard zero measure as a configuration. Let $\Theta$ denote the set of all such configurations in $\mathbb{R}^{d}$. We equip $\Theta$ with the vague topology. $\Theta$ is a Polish space with this topology. (See Prop. 3.17 in $[\mathrm{Re}]$ ). Throughout this paper $\mu$ will denote a probability measure on $(\Theta, \mathfrak{B}(\Theta))$. Of course we are interested in the case such that $\mu\left(\left\{\theta ; \theta\left(\mathbb{R}^{d}\right)=\infty\right\}\right)=1$. We do not a priori assume $\mu$ is a Gibbs measure with a potential $\Phi$.

We introduce a bilinear form related to infinite dynamics $(0.2)$ : Let $\Theta^{i}=$ $\left\{\theta \in \Theta ; \theta\left(\mathbb{R}^{d}\right)=i\right\}$ for $i \in \mathbb{N} \cup\{\infty\}$. Let $\mathbb{R}^{(i)}=\mathbb{R}^{d i}$ for $i \in \mathbb{N}$, and $\mathbb{R}^{(\infty)}=$ $\left\{\left(x_{i}\right)_{1 \leqq i<\infty} ;\left(x_{i}\right)_{1 \leqq i<\infty}\right.$ have no cluster points in $\left.\mathbb{R}^{d}\right\}$. A map $\mathbf{x}^{i}: \Theta^{i} \rightarrow \mathbb{R}^{(i)}$ $(1 \leqq i \leqq \infty)$ is called a $\mathbb{R}^{(i)}$-coordinate of $\theta$ if $\theta=\sum_{j=1}^{i} \delta_{x j}(\theta)$ for all $\theta \in \Theta^{i}$, where $\mathbf{x}^{i}(\theta)=\left(x^{1}(\theta), \ldots, x^{i}(\theta)\right)$. Let for $1 \leqq i \leqq \infty$,

$$
D^{i}[f, g](x)=\frac{1}{2} \sum_{j=1}^{i} \nabla_{j} f(x) \cdot \nabla_{j} g(x) .
$$

Here $\nabla_{j}=\left(\frac{\partial}{\partial x_{j k}}\right)_{1 \leqq k \leqq d}$ and - means the inner product on $\mathbb{R}^{d}$. Let $\mathscr{D}_{\infty}^{\text {loc }}$ be the set of all local, smooth functions on $\Theta$ given by (1.2) in Sect. 1. For $\mathfrak{f}, \mathfrak{g} \in \mathscr{D}_{\infty}^{\text {loc }}$ we set $\mathbf{D}[\mathfrak{f}, \mathfrak{g}]: \Theta \rightarrow \mathbb{R}$ by

$$
\begin{aligned}
\mathbf{D}[\mathfrak{f}, \mathfrak{g}](\theta) & =D^{i}\left[f^{i}, g^{i}\right]\left(\mathbf{x}^{i}(\theta)\right) \text { for } \theta \in \Theta^{i}, 1 \leqq i \leqq \infty \\
& =0 \quad \text { for } \theta \in \Theta^{0} .
\end{aligned}
$$

Here $\mathbf{x}^{i}$ is a $\mathbb{R}^{(i)}$-coordinate, and $f^{i}$ is the permutation invariant function on $\mathbb{R}^{(i)}$ such that $\mathfrak{f}(\theta)=f^{i}\left(\mathbf{x}^{i}(\theta)\right)$ for all $\theta \in \Theta^{i}$. We set $g^{i}$ similarly. Note that such $f^{i}$ and $g^{i}$ are unique for each $i(1 \leqq i \leqq \infty)$ and $\mathbf{D}$ is well defined. We set

$$
\mathscr{E}(\mathfrak{f}, \mathfrak{g})=\int_{\Theta} \mathbf{D}[\mathfrak{f}, \mathfrak{g}](\theta) d \mu, \quad \mathscr{D}_{\infty}=\left\{\mathfrak{f} \in \mathscr{D}_{\infty}^{\text {loc }} \cap L^{2}(\Theta, \mu) ; \mathscr{E}(\mathfrak{f}, \mathfrak{f})<\infty\right\}
$$

Our infinite dynamics are diffusion associated with $\left(\mathscr{E}, \mathscr{D}_{\infty}\right)$ on $L^{2}(\Theta, \mu)$. If one takes $\mu$ as the Poisson random measure on $\mathbb{R}^{d}$ whose intensity measure is Lebesgue 
measure, then $\left(\left(\mathscr{E}, \mathscr{D}_{\infty}\right), L^{2}(\Theta, \mu)\right)$ is associated with $\mathbb{B}_{t}=\sum_{i=1}^{\infty} \delta_{B_{t}^{i}}$, where $\left(B_{t}^{i}\right)_{i \in \mathbb{N}}$ are infinite-many, independent copies of Brownian motion.

Let $Q_{r}=\left\{x \in \mathbb{R}^{d} ;|x| \leqq r\right\}$. Let $Q_{r}^{i}$ be the $i$ times product of $Q_{r}$. We set

$$
\Theta_{r}^{i}=\left\{\theta \in \Theta ; \theta\left(Q_{r}\right)=i\right\} .
$$

We note $\Theta=\sum_{i=0}^{\infty} \Theta_{r}^{i}$. We define $\pi_{r}: \Theta \rightarrow \Theta$ by $\pi_{r}(\theta)=\theta\left(\cdot \cap Q_{r}\right)$. A function $\mathbf{x}: \Theta_{r}^{i} \rightarrow Q_{r}^{i}$ is called a $Q_{r}^{i}$-coordinate (or a coordinate on $\Theta_{r}^{i}$ ) of $\theta$ if

$$
\pi_{r}(\theta)=\sum_{k=1}^{i} \delta_{x^{k}(\theta)}, \quad \mathbf{x}(\theta)=\left(x^{1}(\theta), \ldots, x^{i}(\theta)\right) .
$$
$\mu$ if

Permutation invariant functions $\sigma_{r}^{i}: Q_{r}^{i} \rightarrow \mathbb{R}^{+}$are called density functions of

$\frac{1}{i !} \int_{Q_{r}^{i}} f_{r}^{i} \sigma_{r}^{i} d x=\int_{\Theta_{r}^{i}} \mathfrak{f} d \mu \quad$ for all bounded $\sigma\left[\pi_{r}\right]$-measurable functions $\mathfrak{f}$.

Here $f_{r}^{\imath}: Q_{r}^{i} \rightarrow \mathbb{R}$ is the permutation invariant function such that $f_{r}^{i}(\mathbf{x}(\theta))=\mathfrak{f}(\theta)$ for $\theta \in \Theta_{r}^{i}$, where $\mathbf{x}$ is a $Q_{r}^{i}$-coordinate.

We assume:

$$
\left(\mathscr{E}, \mathscr{D}_{\infty}\right) \text { is closable on } L^{2}(\Theta, \mu)
$$

$$
\sigma_{r}^{i} \in L^{\infty}\left(Q_{r}^{i}, d x\right) \text { for all } i, r, \quad \sum_{i=1}^{\infty} i \mu\left(\Theta_{r}^{l}\right)<\infty \text { for all } r
$$

By (A.1) we denote by $(\mathscr{E}, \mathscr{D})$ the closure of $\left(\left(\mathscr{E}, \mathscr{D}_{\infty}\right), L^{2}(\Theta, \mu)\right)$. Now we state one of our main theorems:

Theorem 1. Suppose that (A.1) and (A.2) hold. Then $(\mathscr{E}, \mathscr{D})$ is a local, quasiregular Dirichlet form on $L^{2}(\Theta, \mu)$.

We refer to $\mathrm{Ma}$ and Röckner $[\mathrm{MR}]$ for the notion of quasi-regularity. Note that $1 \in \mathscr{D}$ and $\mathscr{E}(1,1)=0$. By virtue of Theorem V.2.13, Proposition V.2.15 and Theorem V.1.11 in [MR] we get the following:

Corollary 1. Suppose that (A.1) and (A.2) hold. Then there exists a diffusion $\left\{\mathbb{P}_{\theta}\right\}_{\theta \in \Theta}$ associated with $\left((\mathscr{E}, \mathscr{D}), L^{2}(\Theta, \mu)\right)$. Moreover $\left\{\mathbb{P}_{\theta}\right\}_{\theta \in \Theta}$ is reversible with invariant measure $\mu$.

We reduce (A.1) to a local condition (A.1*), which will be used to check (A.1) for Gibbs measures.

Theorem 2. (A.1) follows from (A.1*):

(A.1 $\left.{ }^{*}\right) \quad\left(\mathscr{E}_{r}^{i}, \mathscr{D}_{\infty}\right)$ are closable on $L^{2}(\Theta, \mu)$ for all $1 \leqq i, r<\infty$,

where $\mathscr{E}_{r}^{i}$ is given by (1.4).

We next consider two types of finite dynamics: Let $\mathscr{E}_{r}=\sum_{i=1}^{\infty} \mathscr{E}_{r}^{i}$ and assume (A.1*). Then by Lemma 2.2 we see $\left(\mathscr{E}_{r}, \mathscr{D}_{\infty} \cap \mathscr{B}_{r}\right)$ and $\left(\mathscr{E}_{r}, \mathscr{D}_{\infty}\right)$ are closable on $L^{2}(\Theta, \mu)$, where $\mathscr{B}_{r}$ is given by $(1.1)$. Let $\left(\mathscr{E}_{r}, \mathscr{D}_{r}\right)$ and $\left(\mathscr{E}_{r}, \widehat{\mathscr{D}}_{r}\right)$ be closures of $\left(\mathscr{E}_{r}, \mathscr{D}_{\infty} \cap \mathscr{B}_{r}\right)$ and $\left(\mathscr{E}_{r}, \mathscr{D}_{\infty}\right)$ on $L^{2}(\Theta, \mu)$, respectively. Then $\left(\left(\mathscr{E}_{r}, \mathscr{D}_{r}\right), L^{2}(\Theta, \mu)\right)$ 
are our finite dynamics; $\left(\left(\mathscr{E}_{r}, \widehat{\mathscr{D}}_{r}\right), L^{2}(\Theta, \mu)\right)$ are finite dynamics studied by Lang. These two sequences of finite dynamics converge to infinite dynamics in the sense of Theorem 3 below.

Let $\mathbb{G}_{r, \alpha}$ and $\mathbb{G}_{\alpha}$ be resolvents of $\left(\left(\mathscr{E}_{r}, \mathscr{D}_{r}\right), L^{2}(\Theta, \mu)\right)$ and $\left((\mathscr{E}, \mathscr{D}), L^{2}(\Theta, \mu)\right)$, respectively. Since $\mathscr{D}_{r}$ is not dense in $L^{2}(\Theta, \mu)$, we define $\mathbb{G}_{r, \alpha}$ in the sense of $[\mathrm{Si}]$. Let $(\widehat{\mathscr{E}}, \widehat{\mathscr{D}})$ denote the closed form on $L^{2}(\Theta, \mu)$ given by $\widehat{\mathscr{D}}=\bigcap_{r=1}^{\infty} \widehat{\mathscr{D}}_{r}$ and $\widehat{\mathscr{E}}(\mathfrak{f}, \mathfrak{f})=\lim _{r \rightarrow \infty} \mathscr{E}_{r}(\mathfrak{f}, \mathfrak{f})$. (See Lemma 2.2.) Let $\widehat{\mathbb{G}}_{r, \alpha}$ and $\widehat{\mathbb{G}}_{\alpha}$ be resolvents of $\left(\left(\mathscr{E}_{r}, \widehat{\mathscr{D}}_{r}\right), L^{2}(\Theta, \mu)\right)$ and $\left((\widehat{\mathscr{E}}, \widehat{\mathscr{D}}), L^{2}(\Theta, \mu)\right)$, respectively.

Theorem 3. (1) $\mathbb{G}_{r, \alpha}$ converge to $\mathbb{G}_{\alpha}$ strongly in $L^{2}(\Theta, \mu)$ as $r \rightarrow \infty$ for all $\alpha>0$.

(2) $\mathbb{G}_{r, \alpha}$ converge to $\widehat{\mathbb{G}}_{\alpha}$ strongly in $L^{2}(\Theta, \mu)$ as $r \rightarrow \infty$ for all $\alpha>0$.

Remark. (1) Suppose $\mu$ is a Gibbs measure in Theorem 4 below. Then there exists diffusions $\left\{\widehat{\mathbb{P}}_{r, \theta}\right\}_{\theta \in \Theta}$ associated with $\left(\left(\mathscr{E}_{r}, \widehat{\mathscr{D}}_{r}\right), L^{2}(\Theta, \mu)\right)$ on $L^{2}(\Theta, \mu) . \quad\left\{\widehat{\mathbb{P}}_{r, \theta}\right\}_{\theta \in \Theta}$ correspond to Lang's ones when $\mu$ satisfies the Lang's assumptions.

(2) $\left(\left(\mathscr{E}_{r}, \mathscr{D}_{r}\right), L^{2}(\Theta, \mu)\right)$ is not quasi-regular because $\left(\left(\mathscr{E}_{r}, \mathscr{D}_{r}\right), L^{2}(\Theta, \mu)\right)$ do not separate the points in $\Theta$. Let $\widetilde{Q}_{r}$ denote the quotient space of $Q_{r}$ identified $\partial Q_{r}=$ $\{|x|=r\}$ with one point. Let $\widetilde{\Theta}_{r}$ denote the set of all configurations on $\widetilde{Q}_{r}$. Then we can regard $\left(\left(\mathscr{E}_{r}, \mathscr{D}_{r}\right), L\left(\Theta, \mu \circ \pi_{r}^{-1}\right)\right)$ as a quasi-regular Dirichlet form on $\widetilde{\Theta}_{r}$. Accordingly, we can construct the associated diffusions on $\widetilde{\Theta}_{r}$.

(3) We will see in Lemma 2.2 that $\mathscr{D} \subset \widehat{\mathscr{D}}$ and $\mathscr{E}(\mathfrak{f}, \mathfrak{f})=\widehat{\mathscr{E}}(\mathfrak{f}, \mathfrak{f})$ for all $\mathfrak{f} \in \mathscr{D}$.

(4) Suppose (L.1) and (L.2) hold. If $\mathscr{D}=\widehat{\mathscr{D}}$, then the distribution of our infinite dynamics $\left\{\mathbb{P}_{\theta}\right\}_{\theta \in \Theta}$ is the same as that of $(0.2)$ obtained by Lang [La1,2]. We however do not know how to prove $\mathscr{D}=\widehat{\mathscr{D}}$. It seems to be related to the explosion of tagged particles; we conjecture that $\mathscr{D}=\widehat{\mathscr{D}}$ holds when all tagged particles have no explosion.

We next give a sufficient condition for (A.1) and (A.2) when $\mu$ is a Gibbs measure with potential $\Phi$ (see Sect. 3 for the definition of Gibbs measure). It is known [Ru2] that for each $z>0$ a Gibbs measure $\mu$ with pair potential $\Phi$ and activity $z$ exists if $\Phi$ satisfies (A.3)-(A.5):

(A.3) $\Phi$ is superstable in the sense of Ruelle [Ru2].

(A.4) $\Phi$ is lower regular in the sense of Ruelle [Ru2]; there exists a positive decreasing function $\phi_{1}$ on $\mathbb{R}^{+}$such that $\Phi(x) \geqq-\phi_{1}(|x|)$ for all $x$, and $\int_{0}^{\infty} \phi_{1}(t) t^{d-1} d t<\infty$.

(A.5) There exist a $R_{1}>0$ and a positive decreasing function $\phi_{2}$ on $\mathbb{R}^{+}$such that $\Phi(x) \leqq \phi_{2}(|x|)$ for $|x| \geqq R_{1}$, and $\int_{R_{1}}^{\infty} \phi_{2}(t) t^{d-1} d t<\infty$.

We will use the following condition to prove (A.1).

(A.6) There exists a $R \in \mathbb{R}$ such that $\Phi$ is finite and upper semicontinuous on $\{|x|>R\}$ and $\Phi(x)=\infty$ on $\{|x| \leqq R\}$.

The constant $R$ in (A.6) is a diameter of hard core particles when $R>0$. If $R<0$, (A.6) means $\Phi$ is upper semicontinuous on $\mathbb{R}^{d}$.

Now we state our second main result:

Theorem 4. Suppose $\Phi$ satisfies (A.3)-(A.6). Then for each $z>0$ there exists a Gibbs measure $\mu$ with pair potential $\Phi$ and activity $z$ satisfying the assumptions in Theorem 1. 
We refer to [Ru1,2] for examples of pair potential $\Phi$ satisfying the conditions in Theorem 4. We note that Lang's conditions (L.1) and (L.2) imply the conditions in Theorem 4.

We now give four examples; the first three examples satisfy the assumptions in Theorem 4, and the last one satisfies ones in Theorem 1. No examples below are covered by Lang's results because their potentials are singular.

To help reader's understanding we give SDEs corresponding to these models. We emphasize that we do not solve these SDEs; we construct diffusions in the category of Dirichlet form theory.

Example 1 (Hard Core Potential). Let $\Phi_{\text {hard }}$ be a hard core potential; $R>0$ and

$$
\Phi_{\text {hard }}(x)=\infty \text { for }|x|<R, \quad \Phi_{\text {hard }}(x)=0 \text { for }|x| \geqq R .
$$

We call $\mu_{\text {hard }}$ a Gibbs measure with hard core interaction if $\mu_{\text {hard }}$ is associated with the potential $\Phi_{\text {hard }}$ and an activity $z>0$. The diffusion obtained by Theorem 1 for $\mu_{\text {hard }}$ describe the motion of infinitely many hard core Brownian balls with diameter $R$.

Example 2 (Lennard-Jones 6-12 Potentials). Let $d=3$ and

$$
\Phi_{6,12}(x)=2 C\left\{|x|^{-12}-|x|^{-6}\right\} \quad(C>0 \text { is a constant }) .
$$

Then $\Phi_{6,12}$ satisfies the conditions in Theorem 4 with $R=0$. See [Ru1,40p]. In this case the corresponding SDE is

$d X_{t}^{i}=d B_{t}^{i}+\sum_{j=1, j \neq i}^{\infty} C\left(X_{t}^{i}-X_{t}^{j}\right)\left\{12\left|X_{t}^{i}-X_{t}^{j}\right|^{-14}-6\left|X_{t}^{i}-X_{t}^{j}\right|^{-8}\right\} d t \quad(1 \leqq i<\infty)$.

Example 3 (Lennard-Jones Type Potentials). Let $a>d$. Set $\Phi_{a}(x)=2|x|^{-a}$. Then $\Phi_{a}$ satisfies the conditions in Theorem 4 with $R=0$. See [Ru1,40p]. In this case the corresponding SDE is

$$
d X_{t}^{i}=d B_{t}^{i}+\sum_{j=1, j \neq i}^{\infty} a\left(X_{t}^{i}-X_{t}^{j}\right)\left|X_{t}^{i}-X_{t}^{j}\right|^{-a-2} d t \quad(1 \leqq i<\infty) .
$$

Example 4 (Dyson's Model). Let $d=1$. Set $\Phi_{D y}(x)=-2 \log |x|$. The corresponding $\mathrm{SDE}$ is

$$
d X_{t}^{i}=d B_{t}^{i}+\sum_{j=1, j \neq i}^{\infty}\left(X_{t}^{i}-X_{t}^{j}\right)^{-1} d t \quad(1 \leqq i<\infty) .
$$

In [Sp2] Spohn studied (0.9) and called this Dyson's model. The potential $\Phi_{D y}$ does not satisfy Ruelle's conditions, so the existence of the associated Gibbs measure does not follow from [Ru2]. He constructed an associated equilibrium measure $\mu$ and obtained correlation functions $\varrho_{r}^{i}$ of $\mu$ explicitly. He proved the existence of the equilibrium dynamics, in the sense of the appropriate Markov semigroup. He however obtained neither the associated diffusion nor the solution of SDE (0.9). We can apply Theorem 1 to this model. Indeed (A.1) follows from Proposition 4 in $[\mathrm{Sp}]$. The correlation functions $\varrho^{i}$ obtained by Spohn are

$$
\varrho^{i}\left(x_{1}, \ldots, x_{i}\right)=\operatorname{det} R_{m, n}, \quad \text { where } R_{m, n}=(2 \pi)^{-1} \int_{|s| \leqq \pi z} e^{i s\left(x_{m}-x_{n}\right)} d s,
$$


where $z$ is a constant. Since correlation functions are bounded, we see $\mu$ satisfies (A.2). We thus construct diffusions associated with $\Phi_{D y}$ by Theorem 1 . We note finite dynamics $\left(\mathscr{E}_{r}, \mathscr{D}_{r}\right)$ for $\Phi_{D y}$ exist because density functions $\sigma_{r}^{i}$ are continuous. We however do not know whether $\left(\mathscr{E}_{r}, \widehat{\mathscr{D}}_{r}\right)$ exist or not.

Now we return to the general situation. One advantage of the Dirichlet form approach is to give a perturbation result. Combining Theorem 1 with the stated results in [MR], we can construct non-symmetric diffusions:

Corollary 2. Let $\left(\mathscr{E}^{*}, \mathscr{D}\right)$ be a (non-symmetric) Dirichlet form on $L^{2}(\Theta, \mu)$ such that

$$
\begin{aligned}
C_{1} \mathscr{E}(\mathfrak{f}, \mathfrak{f}) & \leqq \mathscr{E}^{*}(\mathfrak{f}, \mathfrak{f}) \quad \text { for all } \mathfrak{f} \in \mathscr{D}, \\
\mathscr{E}^{*}(\mathfrak{f}, \mathfrak{g}) & \leqq C_{2} \mathscr{E}(\mathfrak{f}, \mathfrak{f})^{1 / 2} \mathscr{E}(\mathfrak{g}, \mathfrak{g})^{1 / 2} \quad \text { for all } \mathfrak{f}, \mathfrak{g} \in \mathscr{D} .
\end{aligned}
$$

Here $C_{i}(i=1,2)$ are positive constants. Then there exists a diffusion $\left\{\mathbb{P}_{\theta}^{*}\right\}$ associated with $\left(\mathscr{E}^{*}, \mathscr{D}\right)$ with invariant measure $\mu$.

In the forthcoming paper we will apply Corollary 4 to construct diffusions describing infinite many hard core vortexes in viscous planer fluid, and prove a central limit theorem for tagged particles of this model.

A motivation of our work was to construct infinite many hard core Brownian motions in Example 1. We consider this as a reflecting barrier Brownian motion $\mathbb{X}_{t}$ on $\Theta_{R}=\left\{\theta \in \Theta ; \theta=\sum \delta_{x_{l}}\right.$ such that $\left.\left|x_{i}-x_{j}\right| \geqq R\right\}$ and, to some extent, construct this from $\Theta$-valued Brownian motion $\mathbb{B}_{t}$. Recalling the Dirichlet form approach to reflecting barrier Brownian motion on domains in $\mathbb{R}^{d}[\mathrm{~F}]$ [FOT], one may expect that $\mathbb{X}_{t}$ can be constructed from the Dirichlet form $\mathscr{E}(\mathfrak{f}, \mathfrak{g})=\int_{\Theta_{R}} \mathbf{D}[\mathfrak{f}, \mathfrak{g}] d \Lambda$ on $L\left(\Theta_{R},\left.\Lambda\right|_{\Theta_{R}}\right)$. However this idea is formal as it is because $\Lambda\left(\Theta_{R}\right)=0$, unlike the finitely dimensional case. The point is that we can justify this by replacing $\left.\Lambda\right|_{\Theta_{R}}$ by Gibbs measure $\mu_{\text {hard }}$ with hard core potential $\Phi_{\text {hard }}$. This observation is generalized to Theorems 1 and 4.

Dirichlet forms associated with $(0.1)$ have been considered in several papers (see [De, Gu, Sp1, 2].) In these works except [Sp2] the construction of associated processes depends on the solution of $(0.1)$ obtained by Lang. In [Sp1,2] equilibrium fluctuations were studied. In [De, Gu] a central limit theorem for a tagged particle of (0.1) was proved. Recently Tanemura [T] solved SDE (0.1) for hard core potentials.

The organization of this paper is as follows: In Sect. 1 we prepare notations. In Sect. 2 we prove Theorems 1-3. In Sect. 3 we give a definition of Gibbs measures. In Sect. 4 we prove Theorem 4 .

\section{Notations}

In this section we introduce bilinear forms describing finite dynamics.

Let $\pi_{r}^{c}: \Theta \rightarrow \Theta$ be such that $\pi_{r}^{c}(\theta)=\theta\left(\cdot \cap\left\{\mathbb{R}^{d}-Q_{r}\right\}\right)$. For $\mathfrak{f}: \Theta \rightarrow \mathbb{R}$ a function $f_{r, \theta}^{i}(x): \Theta \times Q_{r}^{i} \rightarrow \mathbb{R}$ is called the $Q_{r}^{i}$-representation of $\mathfrak{f}$ if $f_{r, \theta}^{i}$ satisfies the following:

(1) $f_{r, \theta}^{i}(x)$ is a permutation invariant function on $Q_{r}^{i}$ for each $\theta \in \Theta$.

(2) $f_{r, \theta(1)}^{i}(x)=f_{r, \theta(2)}^{i}(x)$ if $\pi_{r}^{c}(\theta(1))=\pi_{r}^{c}(\theta(2)), \theta(1), \theta(2) \in \Theta_{r}^{i}$.

(3) $f_{r, \theta}^{i}\left(\mathbf{x}_{r}^{i}(\theta)\right)=\mathfrak{f}(\theta)$ for $\theta \in \Theta_{r}^{i}$, where $\mathbf{x}_{r}^{i}(\theta)$ is a $Q_{r}^{i}$-coordinate of $\theta$.

(4) $f_{r, \theta}^{i}(x)=0$ for $\theta \notin \Theta_{r}^{i}$. 
Note that $f_{r, \theta}^{i}$ is unique and $\mathfrak{f}(\theta)=\sum_{i=0}^{\infty} f_{r, \theta}^{i}\left(\mathbf{x}_{r}^{i}(\theta)\right)$. When $\mathfrak{f}$ is $\sigma\left[\pi_{r}\right]$-measurable, $Q_{r}^{i}$-representations are independent of $\theta$. In this case we often write $f_{r}^{i}$ instead of $f_{r, \theta}^{i}$. Let $\mathscr{B}_{r}^{*}=\left\{\mathfrak{f}: \Theta \rightarrow \mathbb{R} ; \mathfrak{f}\right.$ is $\sigma\left[\pi_{r}\right]$-measurable $\}$ and $\mathscr{B}_{r}=\left\{\mathfrak{f} \in \mathscr{B}_{r}^{*} ; \mathfrak{f}\right.$ is bounded $\}$. We set

Moreover

$$
\mathscr{B}_{\infty}^{*}=\bigcup_{r=1}^{\infty} \mathscr{B}_{r}^{*}, \quad \mathscr{B}_{\infty}=\bigcup_{r=1}^{\infty} \mathscr{B}_{r}
$$

$$
\mathscr{D}_{\infty}^{\mathrm{loc}}=\left\{\mathfrak{f} \in \mathscr{B}_{\infty}^{*} ; f_{r, \theta}^{i}(x) \text { are smooth on } Q_{r}^{i} \text { for all } i, r, \theta\right\} .
$$

Here $f_{r, \theta}^{i}$ are $Q_{r}^{i}$-representations of $\mathfrak{f}$. It is easy to see

$$
\mathscr{D}_{\infty}^{\mathrm{loc}} \subset C(\Theta)
$$

We will prove in Lemma 2.4 that $\mathscr{D}_{\infty}$ is dense in $L^{2}(\Theta, \mu)$.

For $\mathfrak{f}$ and $\mathfrak{g} \in \mathscr{D}_{\infty}^{\text {loc }}$ we set

$$
\mathbf{D}_{r}^{i}[\mathfrak{f}, \mathfrak{g}](\theta)=D^{i}\left[f_{r, \theta}^{i}, g_{r, \theta}^{i}\right]\left(\mathbf{x}_{r}^{i}(\theta)\right) \quad\left(\theta \in \Theta_{r}^{i}\right), \quad \mathbf{D}_{r}^{i}[\mathfrak{f}, \mathfrak{g}](\theta)=0 \quad\left(\theta \notin \Theta_{r}^{i}\right) .
$$

Here $\mathbf{x}_{r}^{i}(\theta)$ is a $Q_{r}^{i}$-coordinate, and $f_{r, \theta}^{i}$ and $g_{r, \theta}^{i}$ are $Q_{r}^{i}$-representations of $\mathfrak{f}$ and $\mathfrak{g}$, respectively. Note that $\mathbf{D}_{r}^{i}[\mathfrak{f}, \mathfrak{g}]$ is well-defined, that is, it is independent of the choice of $Q_{r}^{i}$-coordinate $\mathbf{x}_{r}^{i}(\theta)$. We now define bilinear forms on $\mathscr{D}_{\infty}$ :

$$
\mathscr{E}_{r}^{i}(\mathfrak{f}, \mathfrak{g})=\int_{\Theta} \mathbf{D}_{r}^{i}[\tilde{\mathfrak{f}}, \mathfrak{g}](\theta) d \mu
$$

\section{Quasi-Regularity of Dirichlet Forms: Proof of Theorems 1, 2 and 3}

In this section we prove Theorems $1-3$. We begin with a monotone convergence theorem on closable forms.

Let $\left\{\left(\mathscr{E}, \mathscr{D}^{n}\right)\right\}$ be a sequence of positive definite, symmetric bilinear forms on $L^{2}(\Theta, \mu)$. We write $\left(\mathscr{E}^{1}, \mathscr{D}^{1}\right) \leqq\left(\mathscr{E}^{2}, \mathscr{D}^{2}\right)$ if

$$
\mathscr{D}^{1} \supset \mathscr{D}^{2} \text { and } \mathscr{E}^{1}(\mathfrak{f}, \mathfrak{f}) \leqq \mathscr{E}^{2}(\mathfrak{f}, \mathfrak{f}) \text { for all } \mathfrak{f} \in \mathscr{D}^{2} \text {. }
$$

We say $\left\{\left(\mathscr{E}^{n}, \mathscr{D}^{n}\right)\right\}$ is increasing if $\left(\mathscr{E}^{n}, \mathscr{D}^{n}\right) \leqq\left(\mathscr{E}^{n+1}, \mathscr{D}^{n+1}\right)$ for all $n$, and we say $\left\{\left(\mathscr{E}^{n}, \mathscr{D}^{n}\right)\right\}$ is decreasing if $\left(\mathscr{E}^{n}, \mathscr{D}^{n}\right) \geqq\left(\mathscr{E}^{n+1}, \mathscr{D}^{n+1}\right)$ for all $n$.

For a given $(\widetilde{\mathscr{E}}, \widetilde{\mathscr{D}})$ with a dense domain $\widetilde{\mathscr{D}}$ we denote by $\left(\widetilde{\mathscr{E}}_{\text {reg }}, \widetilde{\mathscr{D}}_{\text {reg }}\right)$ the largest closable part less than $(\widetilde{\mathscr{E}}, \widetilde{\mathscr{D}})$; that is, $\left(\widetilde{\mathscr{E}}_{\text {reg }}, \widetilde{\mathscr{D}}_{\text {reg }}\right)$ is closable, $\left(\widetilde{\mathscr{E}}_{\text {reg }}, \widetilde{\mathscr{D}}_{\text {reg }}\right) \leqq(\widetilde{\mathscr{E}}, \widetilde{\mathscr{D}})$, and, if $\left(\widetilde{\mathscr{E}}_{*}, \widetilde{\mathscr{D}}_{*}\right)$ is closable and $\left(\widetilde{\mathscr{E}}_{*}, \widetilde{\mathscr{D}}_{*}\right) \leqq(\widetilde{\mathscr{E}}, \widetilde{\mathscr{D}})$, then $\left(\widetilde{\mathscr{E}}_{*}, \widetilde{\mathscr{D}}_{*}\right) \leqq\left(\widetilde{\mathscr{E}}_{\text {reg }}, \widetilde{\mathscr{D}}_{\text {reg }}\right)$. It is known [Si] that the largest closable part exists uniquely.

In the next lemma domains of symmetric forms are not necessary densely defined. So their resolvents are defined in the sense of [Si].

Lemma 2.1. (1) Suppose $\left\{\left(\mathscr{E}^{n}, \mathscr{D}^{n}\right)\right\}$ is increasing. Let $\mathscr{E}^{\infty}(\mathfrak{f}, \mathfrak{f})=\lim _{n \rightarrow \infty} \mathscr{E}^{n}(\mathfrak{f}, \mathfrak{f})$ with the domain $\mathscr{D}^{\infty}=\left\{\mathfrak{f} \in \bigcap_{n} \mathscr{D}^{n} ; \sup \mathscr{E}^{n}(\mathfrak{f}, \mathfrak{f})<\infty\right\}$. Then $\left(\mathscr{E} \infty, \mathscr{D}^{\infty}\right)$ is closable on $L^{2}(\Theta, \mu)$.

(2) In addition to the assumptions of (1), assume $\left(\mathscr{E}^{n}, \mathscr{D}^{n}\right)$ are closed. Then $\left(\mathscr{E} \infty, \mathscr{D}^{\infty}\right)$ is closed and $G_{\alpha}^{n}$ converge to $G_{\alpha}^{\infty}$ strongly in $L^{2}(\Theta, \mu)$ for all $\alpha>0$. 
Here $G_{\alpha}^{n}$ and $G_{\alpha}^{\infty}$ are resolvents of $\left(\mathscr{E}^{n}, \mathscr{D}^{n}\right)$ and $\left(\mathscr{E}^{\infty}, \mathscr{D}^{\infty}\right)$ on $L^{2}(\Theta, \mu)$, respectively.

(3) Suppose $\left\{\left(\mathscr{E}^{n}, \mathscr{D}^{n}\right)\right\}$ is a decreasing sequence of closed forms. Let $\mathscr{E} \infty(\mathfrak{f}, \mathfrak{f})=$ $\lim _{n \rightarrow \infty} \mathscr{E}^{n}(\mathfrak{f}, \mathfrak{f})$ with the domain $\mathscr{D}^{\infty}=\bigcup_{n=1}^{\infty} \mathscr{D}^{n}$. Suppose $\mathscr{D}^{\infty}$ is dense. Then $G_{\alpha}^{n}$ converge to $G_{\alpha, \text { reg }}^{\infty}$ strongly in $L^{2}(\Theta, \mu)$ for all $\alpha>0$. Here $G_{\alpha, \text { reg }}^{\infty}$ are resolvents of the closure of $\left(\mathscr{E}_{\text {reg }}^{\infty}, \mathscr{D}_{\text {reg }}^{\infty}\right)$ on $L^{2}(\Theta, \mu)$ and $\left(\mathscr{E}_{\text {reg }}^{\infty}, \mathscr{D}_{\text {reg }}^{\infty}\right)$ is the largest closable part less than $\left(\mathscr{E}^{\infty}, \mathscr{D}^{\infty}\right)$.

Proof. See [MR, Prop. I.3.7] for (1), and [Si, Theorem 4.1] for (2) and (3).

Lemma 2.2. Let $\mathscr{E}_{r}=\sum_{i=1}^{\infty} \mathscr{E}_{r}^{i}$. Suppose (A.1*) holds. Then

(1) $\left(\mathscr{E}_{r}, \mathscr{D}_{\infty} \cap \mathscr{B}_{r}\right)$ and $\left(\mathscr{E}^{r}, \mathscr{D}_{\infty}\right)$ are closable on $L^{2}(\Theta, \mu)$ for each $r$.

Let $\left(\mathscr{E}_{r}, \mathscr{D}_{r}\right)$ and $\left(\mathscr{E}_{r}, \widehat{\mathscr{D}}_{r}\right)$ denote the closures of $\left(\mathscr{E}_{r}, \mathscr{D}_{\infty} \cap \mathscr{B}_{r}\right)$ and $\left(\mathscr{E}_{r}, \mathscr{D}_{\infty}\right)$, respectively.

(2) $\left\{\left(\mathscr{E}_{r}, \mathscr{D}_{\infty}\right)\right\}_{r \in \mathbb{N}}$ and $\left\{\left(\mathscr{E}_{r}, \widehat{\mathscr{D}}_{r}\right)\right\}_{r \in \mathbb{N}}$ are increasing.

(3) $\left\{\left(\mathscr{E}_{r}, \mathscr{D}_{r}\right)\right\}_{r \in \mathbb{N}}$ is decreasing.

(4) $\lim _{r \rightarrow \infty} \mathscr{E}_{r}(\mathfrak{f}, \mathfrak{f})=\mathscr{E}(\mathfrak{f}, \mathfrak{f})<\infty$ for all $\mathfrak{f} \in \mathscr{D}_{\infty}$.

Proof. By (A.1 $)\left(\sum_{i=1}^{m} \mathscr{E}_{r}^{i}, \mathscr{D}_{\infty}\right)$ are closable. Since $\left\{\left(\sum_{i=1}^{m} \mathscr{E}_{r}^{i}, \mathscr{D}_{\infty}\right)\right\}_{1 \leqq i<\infty}$ is increasing to converge to $\left(\mathscr{E}_{r}, \mathscr{D}_{\infty}\right),\left(\mathscr{E}_{r}, \mathscr{D}_{\infty}\right)$ is again closable by Lemma 2.1 . The closability of $\left(\mathscr{E}_{r}, \mathscr{D}_{\infty} \cap \mathscr{B}_{r}\right)$ follows from that of $\left(\mathscr{E}_{r}, \mathscr{D}_{\infty}\right)$. We thus obtain (1).

Let $\mathfrak{i} \in \mathscr{D}_{\infty}$ and $r<s$. Let $f_{r, \theta}^{i}(x)$ and $f_{s, \theta}^{j}(x)$ be representations of $\mathfrak{f}$ on $\Theta_{r}^{i}$ and $\Theta_{s}^{j}$, respectively. Then for $\theta \in \Theta_{r}^{i} \cap \Theta_{s}^{j}(i \leqq j)$ and a $Q_{r}^{i}$-coordinate $\mathbf{x}_{r}^{i}(\theta)=\left(x_{r}^{1}, \ldots, x_{r}^{i}\right)$, we can choose $Q_{s}^{j}$-coordinate of $\theta$ such that $\mathbf{x}_{s}^{j}(\theta)=\left(x_{r}^{1}, \ldots\right.$, $\left.x_{r}^{i}, x_{s}^{i+1}, \ldots, x_{s}^{j}\right)$. Then for $\theta \in \Theta_{r}^{i} \cap \Theta_{s}^{j}(i \leqq j)$, we see

$$
\sum_{k=1}^{i} \nabla_{k} f_{r, \theta}^{i}\left(\mathbf{x}_{r}^{i}(\theta)\right) \cdot \nabla_{k} f_{r, \theta}^{i}\left(\mathbf{x}_{r}^{i}(\theta)\right) \leqq \sum_{l=1}^{j} \nabla_{l} f_{s, \theta}^{j}\left(\mathbf{x}_{s}^{j}(\theta)\right) \cdot \nabla_{l} f_{s, \theta}^{j}\left(\mathbf{x}_{s}^{j}(\theta)\right) .
$$

Noting $\Theta_{r}^{i} \cap \Theta_{s}^{j}=\emptyset$ if $i>j$, we see

$$
\sum_{i=1}^{\infty} \mathbf{D}_{r}^{i}[\mathfrak{f}, \mathfrak{f}](\theta) \leqq \sum_{i=1}^{\infty} \mathbf{D}_{s}^{i}[\mathfrak{f}, \mathfrak{f}](\theta) \text { for all } \theta \in \Theta .
$$

Integrating both sides we obtain $\left\{\left(\mathscr{E}_{r}, \mathscr{D}_{\infty}\right)\right\}$ is increasing. So its closure $\left\{\left(\mathscr{E}_{r}, \widehat{\mathscr{D}}_{r}\right)\right\}$ is also increasing. This completes the proof of (2).

Let $\mathfrak{f} \in \mathscr{D}_{\infty} \cap \mathscr{B}_{r}$. Then $\sum_{i=1}^{\infty} \mathbf{D}_{r}^{i}[\mathfrak{f}, \mathfrak{f}]=\sum_{i=1}^{\infty} \mathbf{D}_{q}^{i}[\mathfrak{f}, \mathfrak{f}]$ for all $r \leqq q$. Hence $\mathscr{E}_{r}(\mathfrak{f}, \mathfrak{f})=\mathscr{E}_{q}(\mathfrak{f}, \mathfrak{f})=\mathscr{E}(\mathfrak{f}, \mathfrak{f})$. This implies $(3)$ and $(4)$.

Proof of Theorem 2. Theorem 2 follows from Lemma 2.1 (1) and Lemma 2.2 (1), (2), (4).

Proof of Theorem 3. By Lemma 2.1 (2) and Lemma 2.2 (2) we obtain (2). Let $\left(\mathscr{E}_{\text {reg }},\left(\cup \mathscr{D}_{r}\right)_{\text {reg }}\right)$ be the largest closable part less than $\left(\mathscr{E}, \cup \mathscr{D}_{r}\right)$ and $\mathscr{D}_{*}$ its closure. Recall that $\mathscr{D}$ is the closure of $\mathscr{D}_{\infty}$ with respect to $\left(\mathscr{E}, L^{2}(\Theta, \mu)\right)$. By Lemma 2.1 (3) and Lemma $2.2(3)$ it only remains to show $\mathscr{D}_{*}=\mathscr{D}$. By using $\mathscr{E}_{r}(\mathfrak{f}, \mathfrak{f})=\mathscr{E}(\mathfrak{f}, \mathfrak{f})$ for $\mathfrak{f} \in \mathscr{D}_{\infty} \cap \mathscr{B}_{r}$, we obtain

$$
\mathscr{E}_{r}(\mathfrak{f}, \mathfrak{f})=\mathscr{E}(\mathfrak{f}, \mathfrak{f}) \quad \text { for } \mathfrak{f} \in \mathscr{D}_{r} \quad \text { and } \quad \mathscr{D}_{r} \subset \mathscr{D} .
$$


Hence $\cup \mathscr{D}_{r} \subset\left(\cup \mathscr{D}_{r}\right)_{\text {reg }} \subset \mathscr{D}$. So taking closures, we see $\mathscr{D}_{*} \subset \mathscr{D}$. Since $\mathscr{D}_{\infty}=$ $\cup\left(\mathscr{D}_{\infty} \cap \mathscr{B}_{r}\right) \subset \cup \mathscr{D}_{r}$, we see $\mathscr{D}_{\infty} \subset\left(\cup_{\mathscr{D}_{r}}\right)_{\text {reg }}$. Hence we have $\mathscr{D} \subset \mathscr{D}_{*}$. Combining these yields $\mathscr{D}=\mathscr{D}_{*}$.

Lemma 2.3. $\left(\mathscr{E}_{r}, \mathscr{D}_{r}\right),\left(\mathscr{E}_{r}, \widehat{\mathscr{D}}_{r}\right),(\mathscr{E}, \mathscr{D})$ and $(\widehat{\mathscr{E}}, \widehat{\mathscr{D}})$ are Markovian.

Proof. For $\varepsilon>0$ there exists $\varphi_{\varepsilon} \in C^{\infty}(\mathbb{R})$ such that $\varphi_{\varepsilon}(t)=t$ for all $t \in[0,1]$, $\varphi_{\varepsilon}(t) \in[-\varepsilon, 1+\varepsilon]$ and $\left|\varphi_{\varepsilon}^{\prime}(t)\right| \leqq 1$ for all $t \in \mathbb{R}$. Then $\varphi_{\varepsilon} \circ \mathfrak{f} \in \mathscr{D}_{\infty}$ for all $\mathfrak{f} \in \mathscr{D}_{\infty}$, and

$$
\begin{aligned}
& \mathscr{E}_{r}\left(\varphi_{\varepsilon} \circ \mathfrak{f}, \varphi_{\varepsilon} \circ \mathfrak{f}\right) \\
& \quad=\frac{1}{2} \int_{\Theta} \sum_{i=1}^{\infty} \sum_{k=1}^{i}\left\{\varphi_{\varepsilon}^{\prime}\left(f_{r, \theta}^{i}\left(\mathbf{x}_{r}^{i}(\theta)\right)\right)\right\}^{2} \nabla_{k} f_{r, \theta}^{i}\left(\mathbf{x}_{r}^{i}(\theta)\right) \cdot \nabla_{k} f_{r, \theta}^{i}\left(\mathbf{x}_{r}^{i}(\theta)\right) d \mu \\
& \quad \leqq \mathscr{E}_{r}(\mathfrak{f}, \mathfrak{f}),
\end{aligned}
$$

where $f_{r, \theta}^{i}$ is the $Q_{r}^{l}$-representation of $\mathfrak{f}$. This implies $\left(\mathscr{E}_{r}, \widehat{\mathscr{D}}_{r}\right)$ are Markovian. (See Proposition I.4.10 in [MR].) Other statements can be proved similarly.

We next introduce a mollifier on $\mathscr{B}_{\infty}$. Let $j: \mathbb{R}^{d} \rightarrow \mathbb{R}$ be a non-negative, smooth function such that $\int_{\mathbb{R}^{d}} j d x=1$ and $j(x)=0$ for $|x| \geqq \frac{1}{2}$. Let $j_{\varepsilon}=\varepsilon^{d} j(\cdot / \varepsilon)$ and $j_{\varepsilon}^{i}\left(x_{1}, \ldots, x_{i}\right)=\prod_{j=1}^{i} j_{\varepsilon}\left(x_{j}\right)$. For $\mathfrak{f} \in \mathscr{B}_{r}$ we set $\mathfrak{I}_{r, \varepsilon} \mathfrak{f}: \Theta \rightarrow \mathbb{R}$ by

$$
\begin{aligned}
\mathfrak{J}_{r, \varepsilon} \mathfrak{f}(\theta) & =j_{\varepsilon}^{i} * \hat{f}_{r}^{i}(\mathbf{x}(\theta)) \quad \text { for } \theta \in \Theta_{r}^{l}, i \geqq 1 \\
& =\mathfrak{f}(\theta) \quad \text { for } \theta \in \Theta_{r}^{0},
\end{aligned}
$$

where $f_{r, \theta}^{l}(x)=f_{r}^{i}(x)$ is a representation of $\mathfrak{f}$ on $\Theta_{r}^{l}$, and $\hat{f}_{r}^{i}: \mathbb{R}^{d i} \rightarrow \mathbb{R}$ is the function defined by $\hat{f}_{r}^{i}(x)=f_{r}^{i}(x)$ for $x \in Q_{r}^{i}$ and $\hat{f}_{r}^{i}(x)=0$ for $x \notin Q_{r}^{i}$. Moreover $\mathbf{x}(\theta)$ is a $Q_{r}^{i}$-coordinate of $\theta$, and $*$ denotes the convolution. Since $\mathfrak{f}$ is $\sigma\left[\pi_{r}\right]$ measurable, $f_{r, \theta}^{i}$ is independent of $\theta$ and $\mathfrak{f}$ is constant on $\Theta_{r}^{0}$. It is clear that $\mathfrak{I}_{r, \varepsilon} \mathfrak{\uparrow} \in \mathscr{B}_{r}$.

Lemma 2.4. (1) Let $0<\delta<r$ and $\mathfrak{\mathfrak { f }} \in \mathscr{B}_{r-\delta}$. Then we have the following:

$$
\begin{gathered}
\mathfrak{I}_{r, \varepsilon} \mathfrak{f} \in \mathscr{D}_{\infty} \text { for } 0<\varepsilon<\delta, \\
\lim _{\varepsilon \rightarrow 0}\left\|\mathfrak{J}_{r, \varepsilon} \tilde{\mathfrak{f}}-\mathfrak{f}\right\|_{L^{2}(\Theta, \mu)}=0 .
\end{gathered}
$$

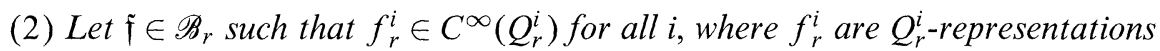
of $\mathfrak{\mathfrak { f }}$. Let $\delta>0$ and $\Theta_{r, \delta}=\left\{\theta \in \Theta ; \theta\left(Q_{r+\delta}-Q_{r}\right)=0\right\}$. Then

$$
\lim _{\varepsilon \rightarrow 0} \mathfrak{J}_{r+\delta, \varepsilon} \mathfrak{f}(\theta)=\mathfrak{f}(\theta) \text { for all } \theta \in \Theta_{r, \delta} \text {. }
$$

(3) $\mathscr{D}_{\infty}$ is dense in $L^{2}(\Theta, \mu)$.

Proof. Let $\mathfrak{g}=\mathfrak{I}_{r, \varepsilon} \mathfrak{f}$, and let $g_{s, \theta}^{j}$ denote its $Q_{s}^{j}$-representation. Since $g_{r, \theta}^{i} \in C^{\infty}\left(Q_{r}^{l}\right)$ for all $i$, we see $g_{s, 0}^{j} \in C^{\infty}\left(Q_{s}^{j}\right)$ for all $s \leqq r$ and $j$. Note that $g$ is $\sigma\left[\pi_{r-\delta+\varepsilon}\right]$ measurable. Hence we also have $g_{s, \theta}^{j} \in C^{\infty}\left(Q_{s}^{j}\right)$ for all $s>r$ and $j$. Combining these we see $\mathfrak{J}_{r, \varepsilon} \mathfrak{f} \in \mathscr{D}_{\infty}^{\text {loc }}$. By this, $\sum_{i=1}^{\infty} i \mu\left(\Theta_{r}^{i}\right)<\infty$ and the property of the mollifier we obtain (2.1).

Note that (2.2) is obvious if $\mu=\Lambda$, where $\Lambda$ is the Poisson random measure whose intensity measure is Lebesgue measure. For each $\delta>0$ there exists an $i$ such that $\mu\left(\bigcup_{j>i} \Theta_{r}^{j}\right) \leqq \delta$. Let $C_{r}^{k}=\left\|\sigma_{r}^{k}\right\|_{L^{\infty}\left(Q_{r}^{k}, d x\right)}$ and $C=\sup \{|\tilde{f}(\theta)| ; \theta \in \Theta\}$. Then 
we see

$$
\left\|\mathfrak{I}_{r, \varepsilon} \mathfrak{f}-\mathfrak{f}\right\|_{L^{2}(\Theta, \mu)}^{2}=\sum_{k=1}^{\infty}\left\|\mathfrak{I}_{r, \varepsilon} \mathfrak{f}-\mathfrak{f}\right\|_{L^{2}\left(\Theta_{r}^{k}, \mu\right)}^{2} \leqq \sum_{k=1}^{i} C_{r}^{k}\left\|\mathfrak{I}_{r, \varepsilon} \mathfrak{f}-\mathfrak{f}\right\|_{L^{2}\left(\Theta_{r}^{k}, \Lambda\right)}^{2}+2 C^{2} \delta .
$$

Combining this with (A.2) yields (2.2). Equation (2.3) is clear by the property of the mollifier. Since $\mathscr{B}(\Theta)=\sigma\left[\pi_{r} ; r \in \mathbb{N}\right], \mathscr{B}_{\infty}$ is dense in $L^{2}(\Theta, \mu)$. Hence (3) follows from (2.2).

Let $\mathbf{A}=\left\{\mathbf{a}=\left\{a_{r}\right\}_{r \in \mathbb{N}} ; a_{r} \in \mathbb{N}, a_{r} \leqq a_{r+1}\right.$ for all $\left.r\right\}$. For $\mathbf{a}=\left\{a_{r}\right\} \in \mathbf{A}$, let $\Theta[\mathbf{a}]=\left\{\theta \in \Theta ; \theta\left(Q_{r}\right) \leqq a_{r}\right.$ for all $\left.r\right\}$. Then $\Theta[\mathbf{a}]$ is a compact set. (See Proposition 3.16 in $[\mathrm{Re}]$.) We introduce a cut off function $\chi[\mathbf{a}]$ of $\Theta[\mathbf{a}]$ as follows:

$$
\chi[\mathbf{a}](\theta)=\rho \circ d_{\mathbf{a}}(\theta), \quad d_{\mathbf{a}}(\theta)=\left\{\sum_{r=1}^{\infty} \sum_{j \in J_{r, \theta}}\left(r-\left|x_{j}(\theta)\right|\right)^{2}\right\}^{1 / 2} .
$$

Here $\left\{x_{j}(\theta)\right\}$ is such that $\theta=\sum \delta_{x_{j}(\theta)},\left|x_{j}(\theta)\right| \leqq\left|x_{j+1}(\theta)\right|$ for all $j$, and

$$
J_{r, \theta}=\left\{j ; j>a_{r}, x_{j}(\theta) \in Q_{r}\right\} .
$$

$\rho: \mathbb{R} \rightarrow[0,1]$ is the function defined by $\rho(t)=1(t<0), \rho(t)=1-t(0 \leqq t \leqq 1)$, and $\rho(t)=0(1<t)$. Note that, if $J_{r, \theta}=\emptyset$ for all $r$, then $d_{\mathbf{a}}(\theta)=0$ and $\chi_{\mathbf{a}}(\theta)=1$.

Let $\|\cdot\|_{1}$ be the norm on $\mathscr{D}$ defined by $\|\mathfrak{f}\|_{1}^{2}=\|\mathfrak{f}\|_{L^{2}(\Theta, \mu)}^{2}+\mathscr{E}(\mathfrak{f}, \mathfrak{f})$.

Lemma 2.5. (1) $\chi[\mathbf{a}]=1$ on $\Theta[\mathbf{a}]$ and $\chi[\mathbf{a}]=0$ on $\Theta\left[\mathbf{a}^{+}\right]^{c}$, where $\mathbf{a}^{+}=\{1+$ $\left.a_{r+1}\right\}_{r \in \mathbb{N}}$.

(2) $\chi[\mathbf{a}] \mathfrak{f} \in \mathscr{D}$ for all $\mathfrak{f} \in \mathscr{D}_{\infty}$.

(3) $\|\chi[\mathbf{a}] \mathfrak{f}\|_{1} \leqq \sqrt{2}\|\mathfrak{f}\|_{1}$ for all $\mathfrak{f} \in \mathscr{D}_{\infty}$.

(4) $\|(1-\chi[\mathbf{a}]) \mathfrak{f}\|_{1} \leqq \sqrt{2 \int_{\Theta[\mathbf{a}]^{c}}\left\{\mathfrak{f}^{2}+\mathbf{D}[\mathfrak{f}, \mathfrak{f}]\right\} d \mu}$ for all $\mathfrak{f} \in \mathscr{D}_{\infty}$.

Proof. (1) is clear by definition. We prove (2) and (3). Let $\chi^{s}[\mathbf{a}]=\rho \circ d_{\mathbf{a}}^{s}$, where $d_{\mathbf{a}}^{s}(\theta)=\left\{\sum_{r=1}^{s} \sum_{j \in J_{r, \theta}}\left(r-\left|x_{j}(\theta)\right|\right)^{2}\right\}^{1 / 2}$. Then $\chi^{s}[\mathbf{a}] \in \mathscr{D}$ and $\lim _{s \rightarrow \infty} \chi^{s}[\mathbf{a}]=\chi[\mathbf{a}]$ in $L^{2}(\Theta, \mu)$. A straightforward calculation shows

$$
2 \mathbf{D}\left[\chi^{s}[\mathbf{a}], \chi^{s}[\mathbf{a}]\right](\theta)=\left\{\frac{\rho^{\prime}\left(d_{\mathbf{a}}^{s}(\theta)\right)}{d_{\mathbf{a}}^{s}(\theta)}\right\}^{2} \sum_{r=1}^{s} \sum_{j \in J_{r, \theta}}\left(r-\left|x_{j}(\theta)\right|\right)^{2}=\rho^{\prime}\left(d_{\mathbf{a}}^{s}(\theta)\right)^{2} \leqq 1 .
$$

Then $\mathscr{E}\left(\chi^{s}[\mathbf{a}], \chi^{s}[\mathbf{a}]\right) \leqq 1 / 2$ for all $s$. We next note that

$$
\begin{aligned}
\mathbf{D}\left[\chi^{s}[\mathbf{a}] \mathfrak{f}, \chi^{s}[\mathbf{a}] \mathfrak{f}\right] & =\mathfrak{f}^{2} \mathbf{D}\left[\chi^{s}[\mathbf{a}], \chi^{s}[\mathbf{a}]\right]+\chi^{s}[\mathbf{a}]^{2} \mathbf{D}[\mathfrak{f}, \mathfrak{f}]+2 \mathfrak{f} \chi^{s}[\mathbf{a}] \mathbf{D}\left[\mathfrak{f}, \chi^{s}[\mathbf{a}]\right] \\
& \leqq 2\left\{\mathfrak{f}^{2} \mathbf{D}\left[\chi^{s}[\mathbf{a}], \chi^{s}[\mathbf{a}]\right]+\chi^{s}[\mathbf{a}]^{2} \mathbf{D}[\mathfrak{f}, \mathfrak{f}]\right\} \\
& \leqq \mathfrak{f}^{2}+2 \mathbf{D}[\mathfrak{f}, \mathfrak{f}]
\end{aligned}
$$

Hence $\sup _{s}\left\|\chi^{s}[\mathbf{a}] \mathfrak{f}\right\|_{1} \leqq \sqrt{2}\|\mathfrak{f}\|_{1}$. Combining these we see $\chi[\mathbf{a}] \tilde{\mathfrak{j}}=\lim _{s \rightarrow \infty} \chi^{s}[\mathbf{a}] \tilde{f}$ weakly in $\left(\mathscr{D},\|\cdot\|_{1}\right)$ and $\|\chi[\mathbf{a}] \mathfrak{f}\|_{1} \leqq \sqrt{2}\|f\|_{1}$. Hence we obtain (2) and (3). The proof of (4) is similar to (3).

Lemma 2.6. There exist $\mathbf{a}_{n}=\left\{a_{n, r}\right\}_{r \in \mathbb{N}} \in \mathbf{A}(n \in \mathbb{N})$ such that $a_{n, r} \leqq a_{n+1, r}$ and that $\mu\left(\bigcup_{n=1}^{\infty} \Theta\left[\mathbf{a}_{n}\right]\right)=1$. 
Proof. Since $\Theta$ is Polish, there exists an increasing sequence of compact sets $K_{n}$ such that $\mu\left(\bigcup_{n=1}^{\infty} K_{n}\right)=1$. It is known (see [Re, Prop. 3.16]) that a subset $A \subset \Theta$ is relatively compact if and only if $\sup _{\theta \in A} \theta\left(Q_{r}\right)<\infty$ for all $r$. Hence $a_{n, r}=$ $\sup _{\theta \in K_{n}} \theta\left(Q_{r}\right)$ satisfy the condition.

For the reader's convenience we give the definition of quasi-regular Dirichlet form. We refer to $[\mathrm{MR}]$ for detail and related notions. A symmetric Dirichlet form $(\varepsilon, F)$ on $L^{2}(\Theta, \mu)$ is called quasi-regular if $(\varepsilon, F)$ satisfies the following:

(Q.1) There exists an $\varepsilon$-nest consisting of compact sets.

(Q.2) There exists an $\|\cdot\|_{1}$-dense subset of $F$ whose elements have $\varepsilon$-continuous $\mu$-versions. Here $\|\mathfrak{f}\|_{1}^{2}=\|\mathfrak{f}\|_{L^{2}(\Theta, \mu)}^{2}+\varepsilon(\mathfrak{f}, \mathfrak{f})$.

(Q.3) There exist $u_{n} \in F, n \in \mathbb{N}$, having $\varepsilon$-continuous $\mu$-versions $\tilde{u}_{n}$, and an $\varepsilon$-exceptional set $N$ such that $\left\{\tilde{u}_{n}\right\}$ separates the points of $\Theta-N$.

Proof of Theorem 1. Let

$$
\mathscr{D}_{0}=\left\{\chi\left[\mathbf{a}_{n}\right] \mathfrak{f} ; \mathfrak{f} \in \mathscr{D}_{\infty}, n \in \mathbb{N}\right\} .
$$

By (2) of Lemma 2.5 we obtain $\mathscr{D}_{0} \subset \mathscr{D}$. By Lemma 2.5 (4) and Lemma 2.6 we see $\mathscr{D}_{\infty} \subset \overline{\mathscr{D}_{0}}$, where - denotes the closure of $\cdot$ with respect to $\|\cdot\|_{1}$. By $\overline{\mathscr{D}_{\infty}}=\mathscr{D}$ we see $\overline{\mathscr{D}_{0}}=\mathscr{D}$. Let $\mathscr{D}(n)=\left\{\mathfrak{f} \in \mathscr{D} ; \mathfrak{f}=0\right.$ a.e. $\theta$ on $\left.\Theta\left[\mathbf{a}_{n}^{+}\right]^{c}\right\}$. By (1) of Lemma 2.5 we see

$$
\mathscr{D}_{0} \subset \bigcup_{n=1}^{\infty} \mathscr{D}(n) .
$$

Hence $\left\{\Theta\left[\mathbf{a}_{n}^{+}\right]\right\}$is a compact nest. We thus obtain (Q.1).

Since $\mathscr{D}_{\infty} \subset C(\Theta)$ and $\mathscr{D}_{\infty}$ is dense in $\mathscr{D},(\mathrm{Q} .2)$ is clear.

Let

$$
U_{r}^{i}=\left\{u \in C^{\infty}\left(Q_{r}^{i}\right) ; u \text { is permutation invariant }\right\} .
$$

We regard elements of $U_{r}^{i}$ as functions on $Q_{r}^{i} / \sim$. Here $\sim$ is the equivalence relation generated by permutations. For each $i, r \in \mathbb{N}$ let $\left\{u_{r, m}^{i}\right\}_{m \in \mathbb{N}}$ be a sequence in $U_{r}^{i}$ that separates the points of $Q_{r}^{i} / \sim$. We can choose $\left\{u_{r, m}^{i}\right\}_{m \in \mathbb{N}}$ so as $i<u_{r, m}^{i}(x) \leqq i+1$ for all $x \in Q_{r}^{i}$. Let $\mathfrak{u}_{r, m}^{i} \in \mathscr{B}_{r}$ be such that $\mathfrak{u}_{r, m}^{i}(\theta)=0$ for $\theta \notin \Theta_{r}^{i}$, and $\mathfrak{u}_{r, m}^{i}(\theta)=$ $u_{r, m}^{i}(\mathbf{x}(\theta))$ for $\theta \in \Theta_{r}^{i}$, where $\mathbf{x}(\theta)$ is a $Q_{r}^{i}$-coordinate of $\theta$. We set $\mathfrak{u}_{r, m}^{0} \equiv 0$ when $i=0$. Then $\left\{\mathfrak{u}_{r, m}^{i}\right\}_{i, r, m \in \mathbb{N}}$ separates the points of $\Theta$.

Let $\theta_{1} \neq \theta_{2} \in \Theta$. Since $\left\{\mathfrak{u}_{r, m}^{i}\right\}_{i, r, m \in \mathbb{N}}$ separates the points of $\Theta$, there exists $(i, r, m)$ such that

$$
\mathfrak{u}_{r, m}^{i}\left(\theta_{1}\right) \neq \mathfrak{u}_{r, m}^{i}\left(\theta_{2}\right) .
$$

Let $0<\delta \in \mathbb{Q}$ such that $\theta_{1}, \theta_{2} \in \Theta_{r, \delta}$, where $\Theta_{r, \delta}=\left\{\theta \in \Theta ; \theta\left(Q_{r+\delta}-Q_{r}\right)=0\right\}$ as before. By (2.3) and (2.7) there exists an $\varepsilon \in \mathbb{Q}$ such that

$$
\mathfrak{J}_{r+\delta, \varepsilon} \mathfrak{u}_{r, m}^{i}\left(\theta_{1}\right) \neq \mathfrak{J}_{r+\delta, \varepsilon} \mathfrak{u}_{r, m}^{i}\left(\theta_{2}\right), \quad 0<\varepsilon<\delta .
$$

which implies $\mathscr{U}=\left\{\mathfrak{I}_{r+\delta, \varepsilon} \mathfrak{u}_{r, m}^{i}\right\}_{i, r, m \in \mathbb{N}, 0<\varepsilon<\delta \in \mathbb{Q}}$ separates the points of $\Theta$. By (2.1) $\mathscr{U}$ is a sequence in $\mathscr{D}_{\infty}$. Hence we obtain (Q.3).

Combining these we see $(\mathscr{E}, \mathscr{D})$ is a quasi-regular Dirichlet form. 
We finally check the local property. Note that the local property is clear for $\left(\mathscr{E}_{r}, \mathscr{D}_{r}\right)$. So we reduce the local property of $(\mathscr{E}, \mathscr{D})$ to that of $\left(\mathscr{E}_{r}, \mathscr{D}_{r}\right)$. Let $\mathfrak{f}$ and $\mathfrak{g} \in \mathscr{D}$ with $\operatorname{supp}[\mathfrak{f}] \cap \operatorname{supp}[\mathfrak{g}]=\emptyset$. Since $\left(\mathscr{E}_{r}, \mathscr{D}_{r}\right)$ is local, we see $\mathscr{E}_{r}(\mathfrak{f}, \mathfrak{g})=0$. Hence $\mathscr{E}(\mathfrak{f}, \mathfrak{g})=\lim _{r \rightarrow \infty} \mathscr{E}_{r}(\mathfrak{f}, \mathfrak{g})=0$, which means $(\mathscr{E}, \mathscr{D})$ is local.

\section{Gibbs Measures with Potential}

In this section we consider the case that $\mu$ is a Gibbs measure with pair potential $\Phi$.

Let $\Phi: \mathbb{R}^{d} \rightarrow \mathbb{R} \cup\{\infty\}$ be a measurable function with $\Phi(x)=\Phi(-x)$. For $x=$ $\left(x^{k}\right) \in Q_{r}^{i}, \theta \in \Theta, r \leqq s$, let

$$
H_{r, \theta, s}^{i}(x)=\sum_{1 \leqq k<l \leqq i} \Phi\left(x^{k}-x^{l}\right)+\sum_{l \in L(r, s)}\left\{\sum_{1 \leqq k \leqq i} \Phi\left(x^{k}-y(\theta)^{l}\right)\right\},
$$

where $\left(y(\theta)^{l}\right)_{l}$ is such that $\theta=\sum_{l} \delta_{y^{l}(\theta)}$ and $L(r, s)=\left\{l ; r<\left|y^{l}(\theta)\right| \leqq s\right\}$. We set

$$
H_{r, \theta}^{i}(x)=\lim _{s \rightarrow \infty} H_{r, \theta, s}^{i}(x), \quad \text { whenever the limit exists . }
$$

Let $Q(z)=Q_{1}+z$, where $z \in \mathbb{Z}^{d}$. Let

$$
\Theta_{0}=\left\{\theta \in \Theta ; \sup _{r \in \mathbb{N}} r^{-d} \sum_{z \in \mathbb{Z}^{d},|z| \leqq r} \theta(Q(z))^{2}<\infty\right\}
$$

It is known (see $[\mathrm{Ru} 2,(5.11)]$ ) that the limit in (3.1) exists for $\theta \in \Theta_{0}$. Let $z>0$ be a number. We set

$$
m_{r, \theta}^{l}(x)=Z_{r, \theta}^{-1} \frac{z^{i}}{i !} \exp \left[-H_{r, \theta}^{i}(x)\right], \quad Z_{r, \theta}=e^{-\left|Q_{r}\right|} \sum_{i=0}^{\infty} \frac{z^{i}}{i !} \int_{Q_{r}^{i}} \exp \left[-H_{r, \theta}^{i}(x)\right] d x .
$$

Here we set the summand to be 1 for $i=0$.

Definition. A probability measure $\mu$ on $(\Theta, \mathscr{B}(\Theta))$ is called a Gibbs measure with potential $\Phi$ and activity $z$ if $\mu$ satisfies the following:

$$
\begin{gathered}
\mu\left(\Theta_{0}\right)=1 \quad \text { (tempered), } \\
\mu\left(A \mid \sigma\left[\pi_{r}^{c}\right]\right)(\theta)=\int_{A} \mathfrak{m}_{r, \theta}\left(\theta_{1}\right) d \Lambda\left(\theta_{1}\right), \quad \text { for } A \in \sigma\left[\pi_{r}\right],
\end{gathered}
$$

where $\pi_{r}^{c}: \Theta \rightarrow \Theta$ such that $\pi_{r}^{c}(\theta)=\theta \cap\left(\mathbb{R}^{d}-Q_{r}\right)$, and $\mathrm{m}_{r, \theta}$ is the $\sigma\left[\pi_{r}\right]$-measurable function defined by $\mathrm{m}_{r, \theta}\left(\theta_{1}\right)=m_{r, \theta}^{i}\left(\mathbf{x}\left(\theta_{1}\right)\right)$ for $\theta_{1} \in \Theta_{r}^{i}$. Here $\mathbf{x}\left(\theta_{1}\right)$ is a $Q_{r}^{i}$-coordinate of $\theta_{1}$.

We similarly define Gibbs measures on finite domains $Q_{s}$ : For $H_{r, \theta, s}^{i}$ we set $m_{r, \theta, s}^{i}$ and $\mathrm{m}_{r, \theta, s}$ similarly as $m_{r, \theta}^{i}$ and $\mathrm{m}_{r, \theta}$, respectively. We define $\mu_{s}$ by $d \mu_{s}=\mathrm{m}_{s, \theta, s} d \Lambda$. Note that $\mathrm{m}_{s, \theta, s}$ is independent of $\theta$.

For $\mu$ and $\mu_{s}$ we define density functions as follows:

$$
\frac{1}{i !} \sigma_{r}^{i}(x)=\int_{\Theta} m_{r, \theta}^{i}(x) d \mu, \quad \frac{1}{i !} \sigma_{r, s}^{i}(x)=\int_{\Theta} m_{r, \theta, s}^{i}(x) d \mu_{s} .
$$


We easily see that $\sigma_{r}^{i}$ are the same as ones given by (0.5). We quote (see Corollary 5.3, Theorem 5.5, Theorem $5.6[\mathrm{Ru} 2])$ :

Lemma 3.1 (Ruelle). Suppose that $\Phi$ satisfies (A.3), (A.4), and (A.5). Then

$$
\sigma_{r, s}^{i}(x) \leqq \xi^{i} \text { for all } i, r, s, \text { where } \xi \text { is a constant } .
$$

Moreover there exists a subsequence $\left\{s_{k}\right\}$ with $s_{k} \uparrow \infty$ and a Gibbs measure $\mu$ with potential $\Phi$ whose density functions $\sigma_{r}^{i}$ and correlation functions $\rho^{i}$ satisfy the following:

$$
\begin{aligned}
& \sigma_{r}^{i}(x)=\lim _{k \rightarrow \infty} \sigma_{r, s_{k}}^{i}(x) \quad \text { uniformly in } x \text { for all } i, r, \\
& \sigma_{r}^{i}(x) \leqq \xi^{i} \text { for all } i, r, \quad \rho^{i}(x) \leqq \xi^{i} \text { for all } i .
\end{aligned}
$$

We will use the following in the proof of Proposition 4.1.

Lemma 3.2. Let $m$ be a nonnegative, bounded, lower semicontinuous function on $Q_{r}^{i}$. Let $\mathscr{E}(f, g)=\int_{Q_{r}^{i}} D^{i}[f, g] m d x$. Then $\left(\mathscr{E}, C_{b}^{\infty}\left(Q_{r}^{i}\right)\right)$ is closable on $L^{2}\left(Q_{r}^{i}, m d x\right)$.

Proof. Let $\mathcal{O}_{n}=\left\{x \in Q_{r}^{i} ; m(x)>1 / n\right\}$, and $\mathscr{E}^{n}(f, g)=\int_{\mathcal{O}_{n}} D^{i}[f, g] m d x$. Then $\left(\mathscr{E}^{n}\right.$, $\left.C_{b}^{\infty}\left(Q_{r}^{i}\right)\right)$ is closable on $L^{2}\left(Q_{r}^{i}, m d x\right)$. Since the sequence $\left\{\left(\mathscr{E}^{n}, C_{b}^{\infty}\left(Q_{r}^{i}\right)\right)\right\}$ of closable forms on $L^{2}\left(Q_{r}^{i}, m d x\right)$ is increasing, its limit $\left(\mathscr{E}, C_{b}^{\infty}\left(Q_{r}^{i}\right)\right)$ is also closable by Lemma 2.1.

\section{Proof of Theorem 4}

In this section we assume $\mu$ is a Gibbs measure with potential $\Phi$ given by Lemma 3.1. The purpose of this section is to prove Theorem 4.

Let $m_{r, \theta}^{i}$ be as in Sect. 3. Let for a.e. $\theta$,

$$
\varepsilon_{r, \theta}^{i}(f, g)=\int_{Q_{r}^{l}} D^{l}[f, g](x) m_{r, \theta}^{i}(x) d x, \quad(f, g)_{r, \theta}^{i}=\int_{Q_{r}^{i}} f(x) g(x) m_{r, \theta}^{i}(x) d x .
$$

Then a straightforward calculation shows for $\mathfrak{f}, \mathfrak{g} \in \mathscr{D}_{\infty}$,

$$
\begin{gathered}
\int_{\Theta} \varepsilon_{r, \theta}^{i}\left(f_{r, \theta}^{i}, g_{r, \theta}^{i}\right) d \mu=\mathscr{E}_{r}^{i}(\mathfrak{f}, \mathfrak{g}), \\
\int_{\Theta}\left(f_{r, \theta}^{i}, g_{r, \theta}^{i}\right)_{r, \theta}^{i} d \mu=\int_{\Theta_{r}^{l}} \mathfrak{f} \mathfrak{g} d \mu,
\end{gathered}
$$

where $f_{r, \theta}^{i}$ and $g_{r, \theta}^{i}$ are $Q_{r}^{i}$-representations of $\mathfrak{f}$ and $\mathfrak{g}$, respectively.

Proposition 4.1. Suppose $\Phi$ satisfies the conditions in Theorem 4. Then for a.e. $\theta,\left(\varepsilon_{r, \theta}^{i}, C_{b}^{\infty}\left(Q_{r}^{i}\right)\right)$ is closable on $L^{2}\left(Q_{r}^{i}, m_{r, \theta}^{i} d x\right)$.

Proof. For $\theta \in \Theta_{0}$ we set $Q_{r, \theta}^{i}=\left\{x=\left(x^{k}\right) \in Q_{r}^{i} ; \quad\left|x^{k}-y^{j}(\theta)\right|>R\right.$ for all $\left.k, j\right\}$. Here $\left\{y^{j}(\theta)\right\}$ is such that $\pi_{r}^{c}(\theta)=\sum_{j} \delta_{y^{j}(\theta)}$, and $R$ is the diameter of hard core given by (A.6). By (A.4), (A.5) and (3.2),

$$
\sum_{j} \sup \left\{\sum_{k=1}^{i}\left|\Phi\left(x^{k}-y^{j}(\theta)\right)\right| ; x=\left(x^{k}\right) \in Q_{r, \theta}^{i}\right\}<\infty \quad \text { for all } \theta \in \Theta_{0} \text {. }
$$


Hence by Lebesgue's convergence theorem $\sum_{j}\left\{\sum_{k=1}^{i} \Phi\left(x^{k}-y^{j}(\theta)\right)\right\}$ is upper semicontinuous and bounded from below on $Q_{r, \theta}^{i}$. We used here (A.6) and the fact that $\Phi$ is bounded from below by (A.3). This implies $m_{r, \theta}^{i}$ is lower semicontinuous and bounded from above on $Q_{r, \theta}^{i}$. Since $m_{r, \theta}^{i}=0$ if $H_{r, \theta}^{i}=\infty$, the same also holds on $Q_{r}^{i}$. Combining this with Lemma 3.2 and (3.3) we complete the proof.

Proof of Theorem 4. (A.2) is clear by (3.8). So we check (A.1*). Let $\{\mathfrak{f}(n)\}$ be an $\mathscr{E}_{r}^{i}$-Cauchy sequence in $\mathscr{D}_{\infty}$ such that $\|\mathfrak{f}(n)\|_{L^{2}(\Theta, \mu)} \rightarrow 0$. Then by (4.1) and (4.2),

$$
\begin{gathered}
\lim _{n \rightarrow \infty} \int_{\Theta}\left\|\mathfrak{f}(n)_{r, \theta}^{i}\right\|_{L^{2}\left(Q_{r}^{l}, m_{r, \theta}^{l} d x\right)} d \mu=0, \\
\lim _{m, n \rightarrow \infty} \int_{\Theta} \varepsilon_{r, \theta}^{i}\left(\mathfrak{f}(m)_{r, \theta}^{i}-\mathfrak{f}(n)_{r, \theta}^{i}, \mathfrak{f}(m)_{r, \theta}^{i}-\mathfrak{f}(n)_{r, \theta}^{i}\right) d \mu=0 .
\end{gathered}
$$

Here $\mathfrak{f}(n)_{r, \theta}^{i}$ are $Q_{r}^{i}$-representations of $\mathfrak{f}(n)$.

We want to prove $\mathscr{E}_{r}^{i}(\mathfrak{f}(n), \mathfrak{f}(n)) \rightarrow 0$. For this purpose it is sufficient to show, for an arbitrary subsequence $\left\{\mathfrak{f}\left(n_{k}\right)\right\}$ of $\{\mathfrak{f}(n)\}$, we can choose a subsequence $\left\{\mathfrak{f}\left(n_{l}\right)\right\}$ of $\left\{\mathfrak{f}\left(n_{k}\right)\right\}$ such that $\mathscr{E}_{r}^{i}\left(\mathfrak{f}\left(n_{l}\right), \mathfrak{f}\left(n_{l}\right)\right) \rightarrow 0$. So let $\left\{\mathfrak{f}\left(n_{k}\right)\right\}$ be an arbitrary subsequence. Then by (4.3) and (4.4) we can choose $\left\{\mathfrak{f}\left(n_{l}\right)\right\}$ in such a way that

$$
\mu\left(A_{l}\right) \leqq 2^{-l}, \quad \mu\left(B_{l}\right) \leqq 2^{-l} .
$$

Here

$$
\begin{aligned}
& A_{l}=\left\{\theta ;\left\|\mathfrak{f}\left(n_{l}\right)_{r, \theta}^{i}\right\|_{L^{2}\left(Q_{r}^{i}, m_{r, \theta}^{l} d \mu\right)} \geqq 2^{-l}\right\}, \\
& B_{l}=\left\{\theta ; \varepsilon_{r, \theta}^{i}\left(\mathfrak{f}\left(n_{l}\right)_{r, \theta}^{i}-\mathfrak{f}\left(n_{l+1}\right)_{r, \theta}^{i}, \mathfrak{f}\left(n_{l}\right)_{r, \theta}^{i}-\mathfrak{f}\left(n_{l+1}\right)_{r, \theta}^{i}\right) \geqq 2^{-2 l}\right\} .
\end{aligned}
$$

By Borel-Cantelli's lemma we see $\mu\left(\lim \sup A_{l}\right)=\mu\left(\lim \sup B_{l}\right)=0$. This means for a.e. $\theta, \lim \left\|\mathfrak{f}\left(n_{l}\right)_{r, \theta}^{i}\right\|_{L^{2}\left(Q_{r}^{i}, m_{r, \theta}^{i} d x\right)}=0$ and $\mathfrak{f}\left(n_{l}\right)_{r, \theta}^{i}$ is an $\varepsilon_{r, \theta}^{i}$-Cauchy sequence. Hence by Proposition 4.1,

$$
\lim _{l \rightarrow \infty} \varepsilon_{r, \theta}^{i}\left(\mathfrak{f}\left(n_{l}\right)_{r, \theta}^{i}, \mathfrak{f}\left(n_{l}\right)_{r, \theta}^{i}\right)=0 \text { for a.e. } \theta .
$$

Recall that $\left\{\nabla \mathfrak{f}\left(n_{l}\right)_{r, \theta}^{i}(x)\right\}$ is $L^{2}\left(Q_{r}^{i} \times \Theta, m_{r, \theta}^{i} d x d \mu\right)$-Cauchy by (4.4) and the definition of $\varepsilon_{r}^{i}$. Combining this with (4.1) and (4.6) implies $\mathscr{E}_{r}^{i}\left(\mathfrak{f}\left(n_{l}\right), \mathfrak{f}\left(n_{l}\right)\right) \rightarrow 0$.

Acknowledgements. The author is thankful to Professor Kusuoka for his valuable comment on convergence of Dirichlet forms describing reflecting barrier Brownian motions. The author is thankful to Professor Tanemura who communicated to the author a mistake on Lemma 2.1 in the first draft.

\section{References}

[De] De Masi, A., Ferrari, P.A., Goldstein, S., Wick, D.W.: An invariance principle for reversible Markov processes. Applications to random motions in random environments. J. Stat. Phys. 55, Nos. 3/4, 787-855 (1989)

[Fr] Fritz, J.: Gradient dynamics of infinite point systems. Ann. Prob. 15, 478-514 (1987)

[F] Fukushima, M.: A construction of reflecting barrier Brownian motions for bounded domains. Osaka J. Math. 4, 183-215 (1967) 
[FOT] Fukushima, M., Oshima, Y., Takeda, M.: Dirichlet forms and symmetric Markov processes. Amsterdam: Walter de Gruyter, 1994

[Gu] Guo, M.Z.: Limit theorems for interacting particle systems. Thesis, Dept. of Mathematics, New York University

[La1] Lang, R.: Unendlich-dimensionale Wienerprocesse mit Wechselwirkung I. Z. Wahrschverw. Gebiete 38, 55-72 (1977)

[La2] Lang, R.: Unendlich-dimensionale Wienerprocesse mit Wechselwirkung II. Z. Wahrschverw. Gebiete 39, 277-299 (1978)

[Lip] Lippner, G.: First order stochastic dynamics of one-dimensional infinite point systems. Point Processes and Queueing Problems. Amsterdam: North-Holland, 1981, pp. 277-289

[MR] Ma, Z., Röckner: Introduction to the theory of (non-symmetric) Dirichlet forms. Berlin, Heidelberg, New York: Springer, 1992

[Re] Resnick, S.I.: Extreme Values, Regular Variation, and Point Processes. Berlin, Heidelberg, New York: Springer, 1987

[Ro] Rost, H.: Non-equilibrium solution of an infinite system of stochastic differential equations. Lecture Notes on Control and Information Sciences 25, Berlin-Heidelberg-New York: Springer, 1980, pp. 297-302

[Ru1] Ruelle, D.: Statistical Mechanics. Rigorous Results., New York: Benjamin, 1969

[Ru2] Ruelle, D.: Superstable interactions in classical statistical mechanics. Commun. Math. Phys. 18, 127-159 (1970)

[Sh] Shiga, T.: A remark on infinite-dimensional Wiener processes with interactions. Z. Wahrschverw. Gebiete 47, 299-304 (1979)

[Si] Simon, B.: A canonical decomposition for quadratic forms with applications to monotone convergence theorems. J. Funct. Anal. 28, 377-385 (1978)

[Sp1] Spohn, H.: Equilibrium fluctuations for interacting Brownian particles. Commun. Math. Phys. 103, 1-33 (1985)

[Sp2] Spohn, H.: Interacting Brownian particles: A study of Dyson's model. In: Hydrodynamic Behavior and Interacting Particle Systems, ed. by G.C. Papanicolaou, IMA Volumes in Mathematics 9, Berlin, Heidelberg, New York: Springer, 1987, pp. 151-179

[T] Tanemura, T.: A system of infinitely many mutually reflecting Brownian balls in $\mathbb{R}^{d}$. Preprint

Communicated by H. Araki 
